\title{
Examining Energy Expenditure in Youth Using XBOX Kinect: Differences by Player Mode
}

\author{
Jourdin Barkman, Karin Pfeiffer, Allie Diltz, and Wei Peng
}

\begin{abstract}
Background: Replacing sedentary time with physical activity through new generation exergames (eg, XBOX Kinect) is a potential intervention strategy. The study's purpose was to compare youth energy expenditure while playing different exergames in single- vs. multiplayer mode. Methods: Participants (26 male, 14 female) were 10 to 13 years old. They wore a portable metabolic analyzer while playing 4 XBOX Kinect games for 15 minutes each (2 single-, 2 multiplayer). Repeated-measures ANOVA (with Bonferroni correction) was used to examine player mode differences, controlling for age group, sex, weight status, and game. Results: There was a significant difference in energy expenditure between single player $(\mathrm{mean}=15.4 \mathrm{ml} /$ $\mathrm{kg} / \mathrm{min}, \mathrm{SD}=4.5)$ and multiplayer mode $($ mean $=16.8 \mathrm{ml} / \mathrm{kg} / \mathrm{min}, \mathrm{SD}=4.7)$. Overweight and obese participants $(\mathrm{mean}=13.7$ $\mathrm{ml} / \mathrm{kg} / \mathrm{min}, \mathrm{SD}=4.2$ ) expended less energy than normal weight (mean $=17.8 \mathrm{ml} / \mathrm{kg} / \mathrm{min}, \mathrm{SD}=4.5$ ) during multiplayer mode $(d=0.93)$. Conclusion: Player mode, along with personal factors such as weight status, may be important to consider in energy expenditure during exergames.
\end{abstract}

Keywords: active video game, exergame, exercise

Children and adolescents are presently recommended to complete at least 1 hour of physical activity of mostly moderate to vigorous intensity every day to live a healthy life. ${ }^{1}$ However, a vast majority of youth do not meet these recommendations because of their sedentary lifestyles. ${ }^{2}$ Screen time is a form of physical inactivity that has been linked to obesity. ${ }^{3}$

One popular form of screen time is playing video games. Electronic games have become extremely popular among young people, with $75 \%$ of adolescents reporting that they play video games on average 3 to 7 times a week. ${ }^{4}$ The new-generation video game consoles that incorporate physical activity by mirroring the players' movements onto the screen offer an opportunity to provide the same video games to which adolescents are accustomed, but with added physical activity. ${ }^{5}$ Previous active gaming systems, such as the Nintendo Wii or the PlayStation Move, resorted to sensors and controllers to simulate movement in the game.

Although Sony was the first to use a camera in their gaming system to capture movement, Microsoft innovated gaming when they used a more technologically advanced camera in their XBOX Kinect system, which allowed for depth perception and automatic camera adjustment. This more advanced image capturing system allowed for a gaming experience with more natural movements. In addition to creating a more natural interactive experience, use of the camera limits the "cheating" that has occurred with previous active gaming systems that allowed for a minimization of effort (eg, flicking the wrist instead of performing an actual movement). ${ }^{5}$ Energy expended from the player could not only be dependent on his/her effort, but it could also be dependent upon type of movement incorporated in the game, different difficulty levels within the game, and most significantly whether the game is being played in multiplayer or single-player mode. ${ }^{6}$ Although one previous study

Barkman (barkmanj@msu.edu), Pfeiffer, and Diltz are with the Dept of Kinesiology; Peng is with the Dept of Media and Information; Michigan State University. compared energy expended during single- vs. multiplayer mode, ${ }^{6}$ the population included in the sample was adults. Thus, it remains unknown if energy expenditure is different in single- vs. multiplayer mode in children and adolescents.

The purpose of the study was to compare the energy expenditure of children and adolescents (10-13 years old) while playing single- vs. multiplayer mode with 4 different video games on the XBOX Kinect.

\section{Methods}

\section{Participants}

A total of 40 adolescents, 26 male and 14 female, 10 to 13 years of age, were recruited through local recreational programs in the mid-Michigan area and emails sent to parent listservs and Michigan State University (MSU) faculty and staff. The study received ethical approval by the MSU Human Research Protection Program. In addition, the parents/guardians and youth, respectively, provided written informed consent and assent to take part in the study. Those participants who refused to sign, were not able to understand consent/assent forms, or had any form of orthopedic limitation that would limit their participation in physical activity were excluded from the study.

\section{Measures}

Anthropometry. Weight was measured to the nearest $0.1 \mathrm{~kg}$ and height was measured to the nearest $0.1 \mathrm{~cm}$ using a scale (Seca 770) and a wall-mounted stadiometer (Harpenden), respectively. Weight was taken while participants were wearing all of the equipment that was used during testing. Shoes were taken off while being measured for height, but left on while being measured for weight. All of the measurements were taken by a trained member of the research team before engaging in the 4 video game sessions. Body mass index $\left(\mathrm{BMI} ; \mathrm{kg} / \mathrm{m}^{2}\right.$ ) was calculated using height and weight, and weight status was assigned according to CDC criteria (www.cdc.gov). 
Energy Expenditure. The Oxycon Mobile portable indirect calorimeter (Cardinal Health, Yorba Linda, CA) was used for energy expenditure assessment. The Oxycon was calibrated within an hour before testing every participant to maintain the highest accuracy possible. In addition, each participant was properly fitted with the correct size mask and vest to minimize any extraneous movement or any leakage of air. Youth were instructed not to eat or drink for at least 2 hours before the testing session. The Oxycon began collecting data at the start of the first 15-minute period and continued until the completion of the final 15-minute period. Expired gases were collected breath-by-breath and were extracted from the system in 10-second increments. Later, the 10-second segments were averaged at the level of the minute for data analyses. The data during the periods of time in-between the testing time that accounted for both rest and manual changes in the XBOX system were not used in analyses. Data were converted to Youth METs (metabolic equivalent) by dividing the energy expenditure by a basal value calculated using the Schofield equation, ${ }^{7}$ which was converted to $\mathrm{ml} / \mathrm{kg} / \mathrm{min}$ using $4.86 \mathrm{kcal} / \mathrm{L} \mathrm{O}_{2}$ and body mass.

Procedures. During each participant's visit, the research team measured height and weight. They also fitted each participant with a Polar heart rate monitor and the metabolic analyzer to collect the participant's expired respiratory gases to estimate energy expenditure.

Participants were then asked to complete 4,15 -minute sessions playing 2 different XBOX Kinect-based games (2 single-player, 2 multiplayer) randomly chosen out of a pool of 4 games. The 4 active video games (Kinect Adventures Reflex Ridge, Just Dance 3, Wipeout, and Kinect Sports Boxing) were chosen because they had produced at least moderate-level physical activity in prior literature. 5,8 The 2 games chosen, the order in which the games were played, and the order in which the participants played single- or multiplayer were randomly decided. If the player completed a level within the 15-minute period s/he was instructed to begin the next most basic level. Before each game, participants received task instructions and were given the opportunity to ask any questions. All games were played on the most basic level provided, intended for beginners. Between games, participants were allowed to remove the mask if they were experiencing discomfort. Upon completion of the visit, the children received $\$ 30$.

\section{Data Analysis}

Data analyses involved examining descriptive statistics and using repeated-measures ANOVA, with Bonferroni adjustment for multiple comparisons, to compare energy expenditure ( $\mathrm{ml} / \mathrm{kg} / \mathrm{min}$ ) between player modes, controlling for age (younger vs. older), sex, weight status (normal weight vs. overweight and obese), and game. Post hoc independent $t$-tests were used to examine betweensubjects effects.

\section{Results}

Descriptive characteristics are in Table 1. Participants were average size for their age and approximated the 65th percentile for BMI. Data for 1 participant were completely removed, and multiplayer values for 2 other participants were removed because of failure of the Oxycon to capture expired gases from the participants due to machine malfunction. The analysis indicated a significant difference between single- and multiplayer modes (within subjects) $[F(1,33)$ $=4.597, P<.05, d=0.498$; Table 2] and a between-subjects effect for weight status $[F(1,33)=9.693, P<.01]$. The post hoc $t$-test revealed that overweight and obese participants (mean $=13.7 \mathrm{ml} / \mathrm{kg} /$ min, $\mathrm{SD}=4.2$ ) had lower energy expenditure for multiplayer mode than normal weight participants [mean $=17.8 \mathrm{ml} / \mathrm{kg} / \mathrm{min}, \mathrm{SD}=4.5$; $t(37)=2.585, P<.05, d=0.927]$. Age group was also a significant covariate, but post hoc tests showed no difference between groups. Youth METs ranged from 3.1 to 4.3 across games and modes.

\section{Discussion}

Results showed higher energy expenditure during multiplayer mode than single-player. These findings are consistent with previous literature regarding player modes. ${ }^{6}$ As previously noted, the prior study included adults, not youth. The current study extends the findings of energy expenditure differences by player mode to youth, since this is the first study to examine player mode in children and adolescents.

Weight status was a significant covariate in analysis and showed that overweight and obese subjects expended less energy during multiplayer mode than normal weight participants. In contrast, Miller et al reported no difference in exergaming energy expenditure by BMI group. ${ }^{9}$ However, their sample had a rather high average BMI, with $50 \%$ of girls and $30 \%$ of boys categorized as overweight or obese. ${ }^{9}$ The participants for the current study had an average BMI in the 65th percentile, which is considered normal weight. Although the current study examined weight status by 2 groups instead of 3, which Miller et al did, Bailey and McInnis found no differences in exergame energy expenditure using 2 groups, either. ${ }^{10}$ Thus, the role of weight status in exergame energy expenditure is not clear.

Some additional factors that could have affected energy expenditure were sex and age of both the player and the opponent. ${ }^{9}$ Sex could potentially play a role regarding the interest in a specific game being played, as well as how the participant played against a second player. In the current investigation, the opponent during multiplayer was not age- or sex-matched. The opponent's gender may have altered the typical way a participant played multiplayer on the XBOX if s/he felt uncomfortable or a different level of challenge based on gender. ${ }^{6}$ However, Miller et al similarly found no sex difference but did find an age difference for exergame energy expenditure. The tight age range and uneven distribution of males

Table 1 Descriptive Characteristics of the Sample (Mean \pm SD)

\begin{tabular}{|c|c|c|c|c|c|c|c|}
\hline & $\mathrm{n}$ & Age (yr) & Height (cm) & Weight (kg) & BMI $\left(\mathbf{k g} \cdot \mathrm{m}^{-2}\right)$ & $\begin{array}{c}\text { BMI } \\
\text { (percentile) }\end{array}$ & $\begin{array}{l}\text { Youth BMR } \\
\text { (kcals/day) }\end{array}$ \\
\hline Total & 40 & $11.0 \pm 0.9$ & $155.0 \pm 10.0$ & $48.0 \pm 12.1$ & $19.5 \pm 3.3$ & $65.4 \pm 23$ & $1441 \pm 204$ \\
\hline Girls & 14 & $10.9 \pm 0.9$ & $154.8 \pm 12.0$ & $48.1 \pm 14.3$ & $19.1 \pm 3.6$ & $63.5 \pm 21.5$ & $1322 \pm 174$ \\
\hline Boys & 26 & $11.0 \pm 0.8$ & $155.2 \pm 9.0$ & $47.9 \pm 11.0$ & $19.8 \pm 3.1$ & $66.4 \pm 24$ & $1505 \pm 192$ \\
\hline
\end{tabular}

Abbreviations: BMR, basal metabolic rate. 
Table 2 Energy Expenditure During Exergame Play in Single- and Multiplayer Modes

\begin{tabular}{|c|c|c|c|c|c|}
\hline Active video game & $\mathrm{ml} / \mathrm{kg} / \mathrm{min}$ & L/min & $\mathrm{kcal} / \mathrm{min}$ & Heart rate & Youth METs \\
\hline \multicolumn{6}{|l|}{ Single-player } \\
\hline Kinect Adventures & $16.6 \pm 3.9$ & $0.75 \pm 0.21$ & $3.6 \pm 1.0$ & $127 \pm 19$ & $3.8 \pm 0.9$ \\
\hline Just Dance 3 & $12.8 \pm 2.8$ & $0.63 \pm 0.17$ & $3.1 \pm 0.8$ & $128 \pm 22$ & $3.1 \pm 0.7$ \\
\hline Wipeout & $17.0 \pm 4.6$ & $0.80 \pm 0.21$ & $3.9 \pm 1.0$ & $128 \pm 32$ & $3.9 \pm 0.9$ \\
\hline Kinect Sports & $14.9 \pm 2.7$ & $0.71 \pm 0.15$ & $3.5 \pm 0.7$ & $128 \pm 24$ & $3.5 \pm 0.6$ \\
\hline \multicolumn{6}{|l|}{ Multiplayer } \\
\hline Kinect Adventures & $18.7 \pm 3.9$ & $0.83 \pm 0.17$ & $4.1 \pm 0.8$ & $133 \pm 15$ & $4.3 \pm 0.7$ \\
\hline Just Dance 3 & $13.2 \pm 3.0$ & $0.64 \pm 0.18$ & $3.1 \pm 0.9$ & $123 \pm 27$ & $3.2 \pm 0.7$ \\
\hline Wipeout & $17.6 \pm 4.5$ & $0.83 \pm 0.20$ & $4.0 \pm 1.0$ & $135 \pm 20$ & $4.1 \pm 0.8$ \\
\hline Kinect Sports & $16.9 \pm 3.6$ & $0.78 \pm 0.33$ & $3.8 \pm 1.6$ & $128 \pm 24$ & $3.7 \pm 1.2$ \\
\hline
\end{tabular}

Abbreviations: MET, metabolic equivalent.

and females may have contributed to the lack of findings in the current study.

In conclusion, the current investigation showed that youth expended more energy in multiplayer than single-player mode and that overweight or obese subjects expended less energy in multiplayer modes than single- player modes. Additional research is necessary to determine if player mode is an influential characteristic of energy expenditure during exergame play in other age groups. Physical activity researchers should take into account potential differences in energy expenditure by player mode and weight status when designing interventions to ensure participants are expending the desired amount of energy.

\section{Acknowledgments}

We wish to acknowledge all of our participants and several undergraduate students at Michigan State University for the time and effort they put into this project. Research was funded by the Department of Media and Information at Michigan State University.

\section{References}

1. U.S. Department of Health and Human Services (USDHHS). 2008 Physical Activity Guidelines for Americans. Washington, DC: USDHHS; 2008.

2. Graves L, Stratton G, Ridgers ND, Cable NT. Energy expenditure in adolescents playing new generation computer games. Br J Sports Med. 2008;42:592-594. PubMed
3. Gao Z, Chen S, Stodden DF. A comparison of children's physical activity levels in physical education, recess, and exergaming. J Phys Act Health. 2015;12:349-354. PubMed doi:10.1123/jpah.2013-0392

4. Allsop S, Rumbold PLS, Debuse D, Dodd-Reynolds C. Real life active gaming practices of 7-11-year-old children. Games Health J. 2013;2:347-353. PubMed doi:10.1089/g4h.2013.0050

5. Rosenberg M, Lay B, Lee M, et al. New-generation active videogaming maintains energy expenditure in children across repeated bouts. Games Health J. 2013;2:274-279. PubMed doi:10.1089/g4h.2013.0037

6. O'Donovan C, Hirsch E, Holohan E, McBride I, McManus R, Hussey J. Energy expended playing XBOX Kinect TM and Wii TM games; a preliminary study comparing single and multiplayer modes. Physiotherapy. 2012;98:224-229. PubMed doi:10.1016/j.physio.2012.05.010

7. Schofield WN. Predicting basal metabolic rate, new standards and review of previous work. Hum Nutr Clin Nutr. 1985;39C:5-41. PubMed

8. Clevenger KA, Howe CA. Energy cost and enjoyment of active videogames in children and teens: XBOX 360 kinect. Games Health J. 2015;4:318-323. PubMed doi:10.1089/g4h.2014.0101

9. Miller TA, et al. Can e-gaming be useful for achieving recommended levels of moderate- to vigorous-intensity physical activity in inner city children? Games Health J. 2013;2(2):96-102. PubMed doi:10.1089/ g4h.2012.0058

10. Bailey BW, Mclnnis K. Energy cost of exergaming a comparison of the energy cost of 6 forms of exergaming. Arch Pediatr Adolesc Med. 2011;165(7):597-602. PubMed doi:10.1001/archpediatrics.2011.15 